Background Best practice guidelines (BPGs) in suicide risk assessment documentation support nursing care of clients at risk for suicide. Investigation regarding nurses' adherence to BPGs for suicide risk assessment documentation is minimal.

Objectives In a mixed-methods study to investigate nurses' knowledge of suicide risk assessment documentation, the researchers created a chart audit to measure nursing practice congruence with five recommendations from the suicide risk assessment BPG (RNAO, 2009).

Methods Five recommendations, from the BPG: Assessment and Care of Adults at Risk for Suicidal Ideation and Behaviour (RNAO, 2009), were the benchmarks for the chart audit measure. Suicide risk indicators, as determined by the Minimum Data Set for Mental Health (MDS-MH) (Ontario Ministry of Health, 2011), were the criteria to identify charts of suicidal clients. The researchers integrated MDS-MH indicators with the five BPG recommendations and constructed compliance indicators that incorporated the Nurses Global Assessment of Suicide Risk (Cutcliffe \& Barker, 2004).

Results Five BPG recommendations, integrated with provincial suicide assessment criteria and a standardised suicide assessment scale produced a 3-point likert scale chart audit with 15 indicators. Possible ranges of scores for documentation congruence with the BPG are 0 to 30 .

Discussion This performance measure provides objective, proxy data to triangulate with nurses' self-perception of suicide risk documentation and evaluate practice as per BPGs.

Implications for Guideline Developers/Users A standardised instrument to monitor BPG practices can be used to inform implementation and education strategies.

\section{P204 THE UPDATING PROCESS GUIDANCE IN CLINICAL PRACTICE GUIDELINES HANDBOOKS: A SYSTEMATIC REVIEW}

1,2,3R Vernooij, 2,3A Sanabria, 2,3 Solà, 2,3 P Alonso-Coello, 2,3 L Martínez García. ${ }^{1}$ Department of Health Sciences, Faculty of Earth and Life Sciences, VU University, Amsterdam; ${ }^{2}$ The Netherlands, Iberoamerican Cochrane Centre, Barcelona, Spain; ${ }^{3}$ Institute of Biomedical Research (IIB Sant Pau), Barcelona, Spain

\section{0:1136/bmjqs-2013-002293.205}

Background Updating is an important process for maintaining recommendations' and clinical practice guidelines' (CPGs) validity. CPGs methodological handbooks are designed to provide guidance on developing and updating CPGs, however, little is known about this guidance about the updating process.

Objectives To identify and describe the guidance about the updating process in CPGs handbooks.

Methods We included methodological handbooks that provide guidance about updating. We conducted a systematic search in the Guidelines International Network library, US National Guidelines Clearinghouse, MEDLINE and contacted main institutions. For extracting data we developed a list of key elements. Results We included thirty-six handbooks. Most of them (97\%) focus mainly on developing de novo CPGs and include some information about updating. Half of the handbooks provide a time frame for updating guidelines. The majority of handbooks do not provide guidance about the updating process, namely: literature search, evidence selection, assessment, synthesis and external review. Finally, two handbooks (6\%) provide information about publishing an updated CPG.

Discussion Our study highlights that the updating process is poorly described in current methodological handbooks.
Handbooks do not contain enough information for executing an optimal update.

Implications Institutions responsible for updating and developing CPGs need to pay more attention to updating and reflect this in their handbooks. This guidance should be more rigorous, explicit and detailed. This could, consequently, lead to a more optimal updating process and, hence, more up-to-date recommendations.

\section{P207 TOOLKIT: IMPLEMENTATION OF BEST PRACTICE GUIDELINES - A FRAMEWORK FOR SUCCESS!}

H McConnell, A Stewart-Pyne, I Bajnok. Registered Nurses' Association of Ontario, Toronto, Canada

\section{0:1136/bmjqs-2013-002293.206}

A signature programme lead by a professional nursing association in Canada has a mandate to develop, disseminate, implement, evaluate and support the uptake of clinical guidelines. This programme's success has resulted in international reach, due to its rigorous guideline development process, and innovative implementation strategies. These strategies include a key resource, the Toolkit: Implementation of Best Practice Guidelines, which delineates a systematic, well-planned implementation process, and is designed to assist nurses and other healthcare professionals to support evidence-informed clinical decisionmaking. This Toolkit is based on emerging evidence that the likelihood of achieving successful uptake of best practice in health care increases when: - Guidelines are selected for implementation through systematic, participatory processes including relevant stakeholder engagement and environmental readiness assessment - Guidelines are tailored to the local context - Barriers and facilitators to guideline use are assessed and addressed - Guideline use is systematically monitored and sustained Evaluation of the impacts of guideline use is an integral part of the entire process - There are adequate resources to support completion of all aspects of implementation This Toolkit will help guideline users take best evidence and integrate it into practice, education and policy using a systematic approach consistent with the local context of practice. This presentation will share the key phases of guideline implementation outlined in the Toolkit, and discuss how this resource is being utilised to address the key challenges of developing evidence based practice cultures through guideline implementation.

\section{P208 HOW COULD WHO BETTER SUPPORT NATIONAL AND SUBNATIONAL GOVERNMENTS IN THEIR EFFORTS TO ADAPT AND IMPLEMENT GLOBAL RECOMMENDATIONS AND DECISIONS? A SYSTEMATIC ANALYSIS OF HEALTH SYSTEMS GUIDANCE AND WORLD HEALTH ASSEMBLY RESOLUTIONS}

${ }^{1,2} \mathrm{D}$ Patino-Lugo, '1 Lavis, ${ }^{3} \mathrm{P}$ Perel, ${ }^{1} \mathrm{Y}$ Wu, ${ }^{3} \mathrm{~A}$ Haines, ${ }^{4} \mathrm{M}$ Ranson, ${ }^{5} \mathrm{U}$ Panisset, ${ }^{6} \mathrm{X}$ Bosch-Capblanch, ${ }^{1} \mathrm{M}$ Brouwers. ${ }^{1} \mathrm{McM}$ aster University, Hamilton, Canada; ${ }^{2}$ University of Antioquia, Medellin, Colombia; ${ }^{3}$ London School of Hygiene and Tropical Medicine, London, UK; ${ }^{4}$ Alliance for Health Policy and Systems Research, WHO, Geneva, Switzerland; ${ }^{5}$ Evidence and Networks for Health - EVIPNet, WHO, Geneva, Switzerland; ${ }^{6}$ Swiss Tropical and Public Health Institute Basel, Switzerland

\section{0:1136/bmjas-2013-002293.207}

Background The World Health Organization's health systems guidance and the normative standards about health systems 